\title{
Time as the Fourth Dimension: Opening up New Possibilities in Chemical Vapor Deposition
}

\author{
Henrik Pedersen
}

\section{Linköping University Post Print}

\section{Tweet}

N.B.: When citing this work, cite the original article.

Original Publication:

Henrik Pedersen , Time as the Fourth Dimension: Opening up New Possibilities in Chemical Vapor Deposition, 2016, Chemistry of Materials, (28), 3, 691-699.

http://dx.doi.org/10.1021/acs.chemmater.5b04553

Copyright: American Chemical Society

http://pubs.acs.org/

Postprint available at: Linköping University Electronic Press

http://urn.kb.se/resolve?urn=urn:nbn:se:liu:diva-125808 


\title{
Time as the fourth dimension:
}

\section{opening up new possibilities in chemical vapor deposition}

\author{
Henrik Pedersen* \\ Department of Physics, Chemistry and Biology, Linköping University, SE-581 83 Linköping, \\ SWEDEN
}

*Corresponding author, Email: henrik.pedersen@liu.se, Twitter: @hacp81 


\begin{abstract}
Thin films of inorganic materials are essential to several technologies we take for granted in our everyday lives. They form the basis of touch screens in smart phones and the electronic components in computers. Dating back more than a century, chemical vapor deposition (CVD) is one of the most common methods to form these films. In CVD, the atoms needed for the thin film are typically supplied by a continuous flow of gaseous precursor molecules and incorporated into the film by gas phase and surface chemical reactions. The continuous demand for more precise thin film fabrication on more complex shapes at lower temperatures sets a demand for more advanced CVD methods. The development of better designed precursor molecules is one important path to evolve CVD, the other path is to evolve the way in which we do CVD. In this perspective I will describe how using time as a fourth dimension in CVD can enable fabrication of new thin film materials and material structures at lower temperatures and on more complex substrate geometries by accessing new types of CVD chemistries available in time-resolved CVD.
\end{abstract}

\title{
Introduction
}

The term chemical vapor deposition (CVD) summarizes a broad class of deposition techniques, many of which are well-established in several industrial areas and used to fabricate thin films for numerous applications ranging from hard coatings to photochromic windows to microelectronics. ${ }^{1}$ Although CVD is in many aspects a mature technique, it needs to evolve in order to meet higher and higher demands on thin films: uniform deposition on more complex geometries, with for example: narrow trenches, on temperature sensitive substrates and onto underlying films which requires lower deposition temperatures. 
Roy Gordon and co-workers summarized controlling the time dimension as an essential characteristic for CVD to be able to deposit conformal thin films in deep features: “conformal CVD reactions must have their slowest, rate-limiting steps on the surface of the growing film, rather than in the gas phase". ${ }^{2}$ That is to say that to realize conformal CVD, the CVD chemistry must be controlled by kinetics instead of thermodynamics. Typically this means doing CVD at lower temperature. From classical CVD theory, a simple Arrhenius plot of the deposition rate vs. the inverse temperature can guide a process to the temperature range where kinetics controlled deposition chemistry occurs. Angel Yanguas-Gil, John Abelson and co-workers highlighted the importance of temperature for conformal CVD: “At sufficiently high gas temperature and pressure, most precursor molecules will undergo gas phase reactions; indeed this is required in some CVD systems to generate the desired growth species. However, the conformal coverage regime largely occurs at lower temperature". ${ }^{3}$ Thus, we can see that low temperature CVD is desired not only from a substrate tolerance perspective, but also from a conformity perspective. However, temperature is not the only parameter controlling CVD kinetics, the total pressure of the system and partial pressure of the precursor are also of great importance. Additionally, conformal, surface controlled CVD can also be achieved by allowing only one of the precursors to react with the surface at the time, which is the principle for the highly successful form of CVD known as atomic layer deposition (ALD). Typically the temperature must also be lowered for a successful ALD process, since ALD depends on a surface-saturating chemistry where chemisorption is followed by stabilization, while a too high of a temperature will lead to a surface-activated chemistry where chemisorption is followed by controlled decomposition of the precursor molecules.

In this perspective paper I will discuss some new possibilities - not only conformal and low temperature CVD - available when CVD is done in a time-resolved rather than continuous manner. I will point to how both low temperature, kinetics control and favorable pressure 
regimes are accessible when controlling the time dimension of CVD. First I will discuss the approach of time-resolved CVD where the precursor molecules are separated in time by introducing them in alternating pulses, eliminating certain chemical reactions by preventing the molecules from making contact and enabling other less favored reactions. To date, this is the most explored time-resolved CVD approach. I will then discuss different time-resolved plasma CVD routes where either gas flow or energy supply can be time-resolved to access new CVD chemistries.

\section{Time-resolved precursor supply in CVD}

Among all CVD techniques, ALD is one of the most high-lighted as it has proven essential in the fabrication of today's nanoscale electronics. ${ }^{4}$ Its success lies in the fact that it is a timeresolved CVD technique - the continuous flow of a mixture of precursors diluted in a carrier gas in conventional CVD is - in ALD - replaced by a sequence of precursor pulses separated in time by inert gas pulses. Thus, in ALD, the substrate surface is exposed to only one of the precursor molecules at a time and the inert gas pulses make sure that any reactions between the precursors are avoided (Fig. 1). The field of ALD has been reviewed extensively several times, Refs. 5, 6, 7, 8, 9 and 10 are recommended for further reading on ALD.

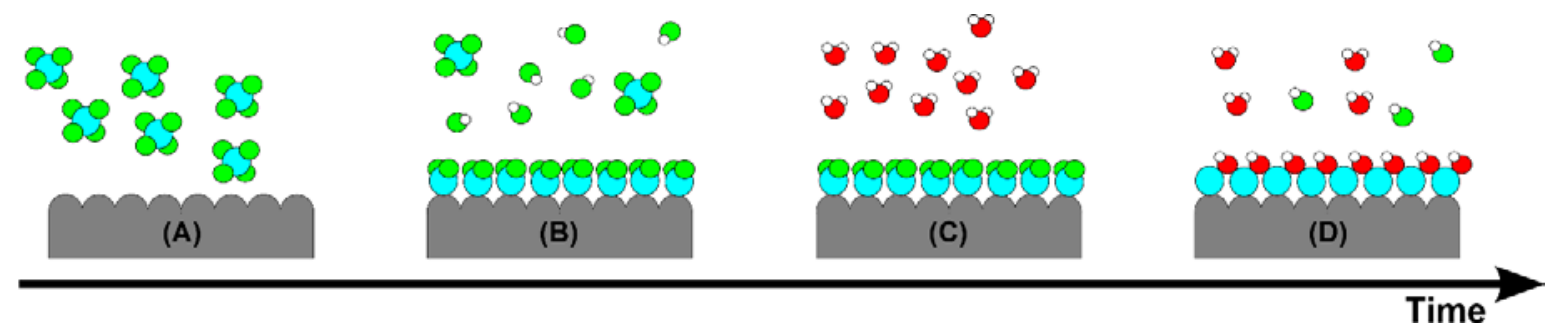

Figure 1. Schematic of film deposition by atomic layer deposition. As the substrate is exposed to the first precursor (A), a monolayer forms by chemisorption of the first precursor 
(B). The monolayer needs to be at least kinetically stable while unreacted precursors and byproducts are purged away and the substrate is exposed to the next precursor (C). The second precursor reacts to form a layer of the material (D) which again needs to be at least kinetically stable until the first precursor is introduced in the next ALD cycle.

A successful ALD process uses precursors which are thermally stable in the gas phase and react only in contact with a surface. In the classic ALD model, the precursors, once chemisorbed on the substrate, must also be thermally stable on the substrate, ${ }^{11}$ meaning that the process must form a thermodynamically stable monolayer. In other words, ALD should have a surfacesaturating chemistry with chemisorption followed by stabilization. However, we include the dimension of time in ALD and carefully adjust the pulse and purge times to a range where the adsorbed monolayer is kinetically stable, if thermodynamic stability is not possible. That is to say that we need to tune the kinetics so that surface-activated CVD chemistry where controlled decomposition after the chemisorption is sufficiently delayed. If we adopt this view of ALD we could start to consider kinetics as a precursor in ALD, and thereby using the time dimension more fully. As long as the monolayer is stable - kinetically or thermodynamically - until the next precursor is introduced, the deposition chemistry is self-limiting: once the surface is saturated by precursors, no further deposition occurs before the next precursor pulse. The selflimiting deposition chemistry of ALD allows for perfectly conformal deposition of thin films on surfaces with a very complex topology, which is vital for the fabrication of today's microand nano-electronics ${ }^{12}$ and a high degree of in-situ monitoring, ${ }^{13}$ not accessible for continuous CVD.

Depositions that do not lead to a self-limiting deposition chemistry should not be labeled as ALD since self-limiting deposition is the very essence of ALD. The precursor pulsing can, however, still result in interesting processes and the terms "pulsed CVD" or "precursor 
pulsed/modulated CVD” is often used to highlight time-resolved gas delivery. One particularly interesting effect of precursor pulsing which did not result in a self-limiting deposition was the discovery that nanowires of GaN could be grown without metal catalysts on substrates with a patterned silicon nitride mask by pulsing the standard GaN CVD precursors trimethyl gallium $\left(\mathrm{Ga}\left(\mathrm{CH}_{3}\right)_{3}\right)$ and ammonia $\left(\mathrm{NH}_{3}\right){ }^{14}$

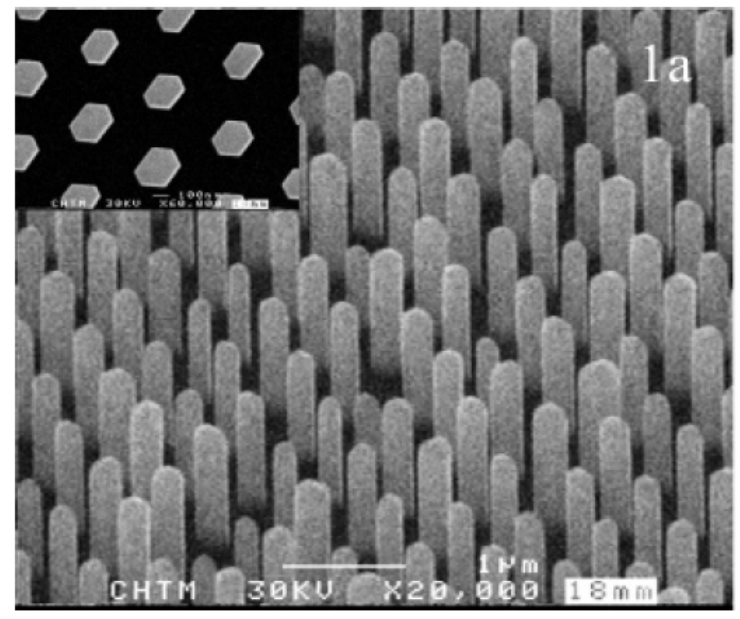

Figure 2. GaN nanowires grown without use of catalyst by precursor pulsed CVD using a patterned silicon nitride mask on the substrate. Insert shows the hexagonal symmetry of the wires in top view, the symmetry emanates from the hexagonal pattern of the mask. Reprinted with permission from Ref. 14. Copyright 2006 American Chemical Society.

The resulting nanowires (Fig. 2) show hexagonal symmetry that results from the pattern fabricated by lithography in the silicon nitride mask. The $30 \mathrm{~nm}$ thick silicon nitride mask was deposited by CVD at low pressure on GaN grown on sapphire, silicon or silicon carbide. The hexagonal holes were etched through the mask: as $\mathrm{GaN}$ does not nucleate on $\mathrm{SiN}_{\mathrm{x}}$, the $\mathrm{GaN}$ continuous-mode CVD done after lithography resulted in deposition only in the hexagonal holes. Continuous CVD after the holes were filled led to lateral overgrowth and eventually a continuous film. But by pulsing the precursors after filling of the holes led to a controlled growth of nanowires whose diameter (set by the diameter of the holes in the mask) was constant 
even when the wires grew to several $\mu \mathrm{m}$ in length. The authors used a precursor pulsing scheme of $20 \mathrm{~s} \mathrm{Ga}\left(\mathrm{CH}_{3}\right)_{3}$ and $30 \mathrm{~s} \mathrm{NH}$, i.e. significantly longer pulsed times compared to those commonly used in ALD, and pointed out that this scheme most likely has to be re-optimized when transferring this process to other CVD reactors. ${ }^{14}$ The nanowires grew in the c-direction, i.e. on the (0001) surface, with $\{1-102\}$ facets. The sides of the wires were $\{1-100\}$ facets. In their study the authors found that during precursor pulsed CVD, the ratio of vertical to lateral growth rate was higher than 1000, i.e. growth rate on the (0001) plane and on the $\{1-102\}$ facets is 1000 times faster than the growth rate on the $\{1-100\}$ facets. This immense difference exisated despite the much larger area of the sides. The authors then speculated that the low effective $\mathrm{N} / \mathrm{Ga}$ ratio that occurred during the $\mathrm{Ga}\left(\mathrm{CH}_{3}\right)_{3}$ pulse preferentially destabilized the less stable facets at the tip of each nanowire to allow for a vapor-liquid-solid (VLS) type of growth. The authors further suggested that liquid gallium would act as the catalyst in such a VLS mechanism. This paper is a beautiful demonstration of how time-resolved CVD truly opens up new possibilities of making materials.

Time-resolved precursor delivery can also be used to deposit thin films of ternary or quaternary materials. By combining known CVD and ALD chemistries for $\mathrm{Cu}_{3} \mathrm{~N}$ and $\mathrm{Ni}_{3} \mathrm{~N}$ in a sequential manner separated by $\mathrm{NH}_{3}$ pulses a ternary solid solution of $\mathrm{Cu}_{3-\mathrm{x}} \mathrm{Ni}_{\mathrm{x}+\mathrm{y}} \mathrm{N}$ can be deposited. ${ }^{15} \mathrm{In}$ contrast, no deposition at all was obtained when using a non-pulsed CVD approach where all precursors were mixed together. This is speculated to be due to mutual blocking of the surface chemical reactions by the ligands or fragments of the ligands of the metal precursors; $\mathrm{Cu}(\mathrm{hfac})_{2}$ and $\mathrm{Ni}(\text { thd })_{2}$. The $\mathrm{NH}_{3}$ pulse separating the copper nitride and nickel nitride CVD chemistries in time was believed to serve as a surface cleaning step rendering a $-\mathrm{NH}_{\mathrm{x}}$ terminated surface, allowing deposition of the next metal nitride. This surface cleaning also led to very low contamination levels in the films. A few monolayers of each metal nitride was deposited during each CVD sequence. The metal nitrides were then mixed by diffusion to allow $\mathrm{Ni}$ atoms to fill 
the low space-filling $\mathrm{Cu}_{3} \mathrm{~N}$ crystal structure to form the ternary solid solution. The authors further described how the $\mathrm{Cu} / \mathrm{Ni}$ ratio can be varied by changing the gas pulsing sequence and propose that this time-resolved CVD concept should permit the synthesis of many complex material systems.

\section{Time-resolved pressure in CVD}

A different time-resolved CVD approach that, like ALD, uses pulsed precursor flows to achieve a higher thickness uniformity over large objects with complex geometry is pulsed pressure CVD where pulsed precursor injection is used to vary the pressure over time (Fig 3).

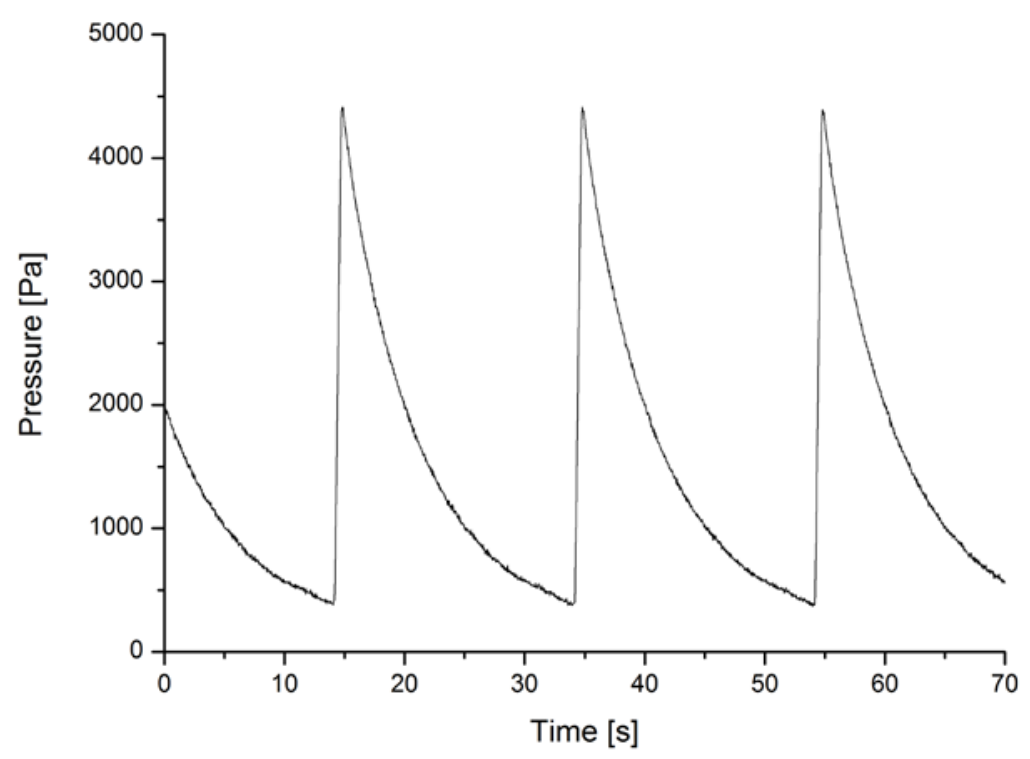

Figure 3: Variation of reactor pressure over time in pulsed pressure CVD during operation. Plotted from data generously provided by Prof. Susan Krumdieck, University of Canterbury, New Zealand. A similar figure has previously been published in Ref. 16.

This approach to CVD is significantly different from standard CVD in that it does not use a carrier gas to transport the volatile precursor. Instead, the precursor was dissolved in a liquid solvent and the solution was injected via an ultrasonic atomizing nozzle, directly into a 
continuously evacuated deposition chamber in discreet timed injections. In the chamber, flash evaporation of the atomized droplets led to a sharp spike in pressure that was instantly decreased by the continuous vacuum. The precursor solution delivery is crucial in this CVD approach. The injection of the solution must be done rapidly so that all droplets are exposed to the lowest chamber pressure. If injection continues while the chamber pressure is increased, droplets entering the vacuum chamber at a later time - exposed to a higher pressure - will be more likely to undergo continuous solvent evaporation, which can lead to formation of an aerosol of the precursor. Furthermore, if the droplets that enter the chamber are too large, they survive for even a short time before flash evaporation and will also likely dry out to form an aerosol of dried precursor. Thus, smaller droplets and very rapid injection are desired. The solvent used should have a high vapor pressure, low surface tension and low viscosity to increase the vaporization efficiency. However, chemical compatibility of the solvent with the particular precursor being used is the prime consideration. The precursor concentration in the solvent is also quite important. Higher concentration increases deposition rate since more precursor is injected in each pulse. However, a higher concentration solution also decreases vaporization efficiency because of a greater tendency to form aerosols during evaporation. ${ }^{17}$

Pulsed pressure CVD was first reported in 1993 as a CVD route to the complex metal oxide $\mathrm{LiTaO}_{3 .}$, using metal alkoxides as single precursor dissolved in toluene for both oxides. ${ }^{18}$ Since then, the main body of the studies on pulsed pressure CVD has been carried out on $\mathrm{TiO}_{2}$ (first reported 1995). ${ }^{19}$ A significant advantage with the pulsed pressure CVD technique is that the pressure pulsing gives access to both a laminar gas flow when the pressure is higher and a molecular gas flow when the pressure is lower. ${ }^{20}$ It is well known in CVD that low pressure CVD gives a higher uniformity, total pressure and precursor partial pressure are also pointed out as key factors for conformal CVD. ${ }^{3}$ This can be used to achieve a conformal deposition: very good conformity has been demonstrated on $100 \mathrm{~nm}$ sized features with aspect ratios 2:1.20 
This conformity is not impressive if compared to ALD, but the deposition rate in pulsed pressure CVD is on the order of $2 \mathrm{~nm}$ per pulse, compared with $0.05-0.1 \mathrm{~nm}$ per pulse in ALD. Apart from $\mathrm{LiTaO}_{3}$ and $\mathrm{TiO}_{2}$, yttria-stabilized zirconia, $\mathrm{ZrO}_{2}-\mathrm{Y}_{2} \mathrm{O}_{3}$ (YSZ) and hydroxyapatite, $\mathrm{Ca}_{10}\left(\mathrm{PO}_{4}\right)_{6}(\mathrm{OH})_{2}$ have also been reported deposited by pulsed pressure CVD on solid oxide fuel cell electrodes ${ }^{21}$ and tantala bone scaffolds ${ }^{22}$ respectively. The pulsed pressure CVD process has also been up scaled for coatingcomplex objects on the tens of centimeter scale, such as stainless steel pump impellers. ${ }^{17}$

The development of ALD valves allows for gas switching on the millisecond scale ${ }^{23}$ and sets the time resolution of the gas mixture in ALD, and thereby also CVD, to the millisecond range. Pulsed pressure CVD also uses pulsing of precursors to resolve the process in time, although the pulsing is done to rapidly change the gas pressure in the chamber to access different types of gas flow behavior. Thus far only thermally activated CVD processes has been considered, and since pulsing temperature on a large thermal budget is a very slow process this cannot be exploited to achieve a CVD process time-resolved in the aspect of energy supply. To be able to introduce time as a fourth dimension in the energy supply to CVD, one option is to use the energy in a plasma to activate CVD chemistry.

\section{The plasma state - a chemical playground for CVD}

An alternative to elevated temperatures for supplying the energy needed to overcome the activation energy for chemical reactions, CVD can also be activated by energy in other forms, most notably in the form of electric energy stored in a plasma. The main motivation for using a plasma in CVD is to have a low substrate temperature, but the lower overall temperature for forming essentially the same thin film means that plasma CVD is done much further away from thermodynamic equilibrium. Chemical reactions are all about electron transfer between atoms and molecules: the abundance of free electrons makes plasma a chemical playground where 
new reaction pathways using the free electrons are accessible. The plasma chemical reactions of interest for CVD are summarized in Fig. 4 drawn from Ref. (24). Depending on their energy, plasma electrons can excite, dissociate or even ionize the precursor molecule, $\mathrm{X}_{2}$. Plasma CVD can therefore activate an inert molecule such as $\mathrm{N}_{2}$ to be a CVD precursor even at low deposition temperature. Also excited neutral and positive ionic plasma species, $\mathrm{A}^{*}$ and $\mathrm{A}^{+}$ respectively, in Fig. 4, can dissociate and ionize precursor molecules. Finally recombination reactions where ionized precursor species combine with electrons or dissociated precursor species are also possible. Similar to thermal CVD, a boundary layer will develop over the substrate when a gas mixture flows over it and thus a neutral species must diffuse through the boundary layer to reach the substrate surface. In addition to the boundary layer, a plasma sheath will develop over the substrate surface and all other surfaces near the plasma. The plasma sheath is the layer in which the electrical potential of the plasma drops to the electrical potential of the surface in contact with the plasma. The thicknesses of both the boundary layer and the plasma sheath decreases as the total pressure in the CVD process decreases. Due to the potential drop, charged species with the opposite charge relative to the substrate will be accelerated in the plasma sheath toward the substrate. This creates an ion bombardment of the surface that acts as an additional source of energy to surface processes and can be used to enhance desorption of byproducts. Surface bombardment can also play a crucial role in obtaining a crystalline film but can also create problematic stress in the film. ${ }^{25}$ 


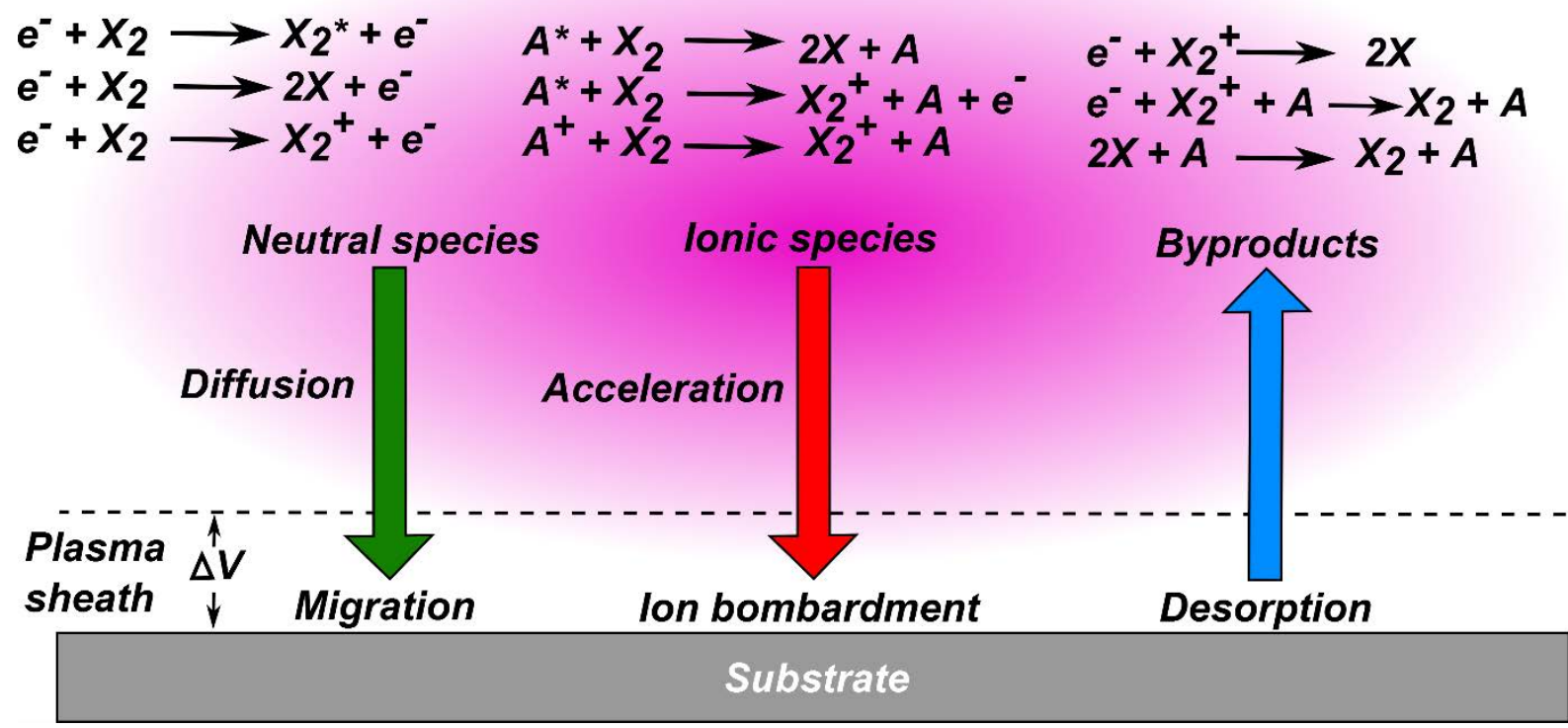

Figure 4: Summary of the plasma chemical reactions important for plasma CVD, adapted from Ref 24. $\mathrm{X}_{2}$ denotes a diatomic CVD precursor and A denotes plasma gas species. Note that the reactions are not limited to diatomic precursor molecules.

This becomes a another dimension in time-resolved CVD: plasma allows rapid and abrupt change in the input of energy to the chemical reactions over time, on a much shorter time scale and in a much more precise way than a thermal based process would.

\section{Time-resolved precursor supply in plasma CVD}

The successful time-resolved CVD technique ALD, described above, has taken a somewhat new direction with the addition of a plasma discharge to the ALD cycle. The difference between thermally activated ALD and plasma ALD is the introduction of a plasma discharge typically in the second precursor pulse, Fig. 5. Plasma ALD is therefore time-resolved both in precursor supply and in plasma discharge. The first half of the ALD cycle is the same for both thermal ALD and plasma ALD: the substrate is subjected to metal precursor molecules which chemisorb 
and form a monolayer which needs to be at least kinetically stable until a plasma, typically a $\mathrm{O}_{2}, \mathrm{~N}_{2}$ or $\mathrm{H}_{2}$ plasma, is used as the second precursor. This plasma gas which provides the radicals for the second reaction step is, however, not delivered in a time-resolved manner as it is not reactive with the metal precursor. Plasma ALD uses reactive species from plasma to oxidize, nitridize or reduce the surface and remove the ligands from the first precursor. Typically $\mathrm{O}^{\bullet}$ and $\mathrm{N}^{\bullet}$ radicals from $\mathrm{O}_{2}$ and $\mathrm{N}_{2}$ plasmas are used to form oxides and nitrides, respectively. $\mathrm{H}_{2}$ plasmas are used as reducing agents when depositing metallic films. The field of plasma ALD has been extensively reviewed in Ref. 26 where a list of thin films deposited by plasma ALD is also given.

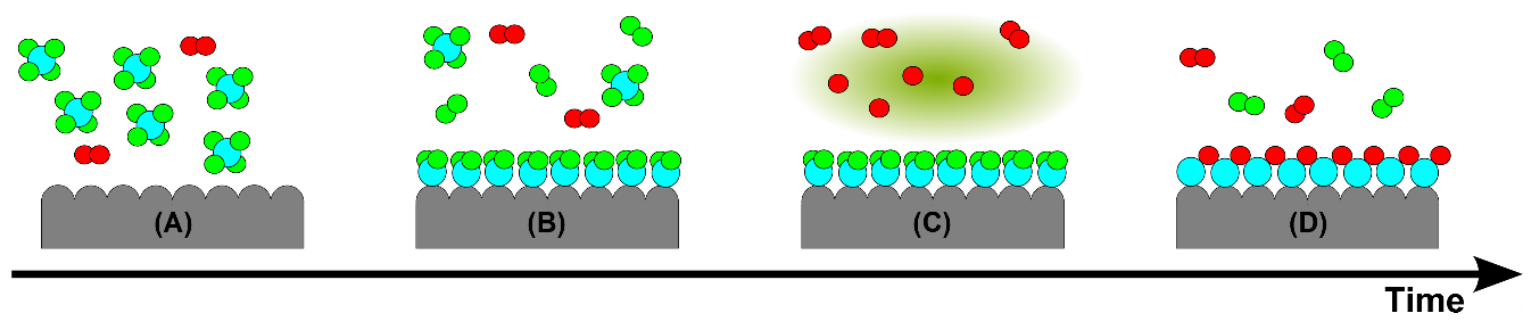

Figure 5: Schematic of film deposition by plasma ALD. As in thermal ALD, the substrate is exposed to the first precursor (A) and a monolayer forms by chemisorption of the first precursor (B). The monolayer needs to be at least kinetically stable while unreacted precursors and byproducts are purged away. The plasma gas (red molecules) is not pulsed in time but it is only when the plasma is ignited (C) that the plasma gas form reactive species which reacts to form a layer of the material (D) which again needs to be at least kinetically stable until the first precursor is introduced in the next ALD cycle.

The use of a plasma in ALD allows for significantly lower deposition temperatures compared to thermal ALD: While thermal ALD typically is done at 200-600 ${ }^{\circ} \mathrm{C}$, plasma ALD has been reported to produce $\mathrm{Al}_{2} \mathrm{O}_{3}, \mathrm{TiO}_{2}$ and $\mathrm{SiO}_{2}$ films at room temperature. ${ }^{27}$ Room temperature plasma ALD has further been extended to metallic platinum films, where it was necessary to 
first use an oxygen plasma to remove the ligands from the Pt precursor and then a hydrogen plasma to reduce the formed $\mathrm{PtO}_{\mathrm{x} .}{ }^{28}$ This route was successfully used to deposit Pt films on polymers, textiles and paper showing the potential to use a low temperature plasma ALD route on temperature sensitive substrate materials.

The main hurdle for plasma ALD is to obtain the highly conformal films that are the hallmark of (thermal) ALD. ${ }^{29}$ The problem lies in the very nature of plasma ALD: the use of and dependence on very reactive plasma generated radicals for the surface reactions. The reactive species will not diffuse down to the bottom of narrow features before reacting with the surface. Diffusion in a narrow feature depends on non-reactive collisions with the surface - well designed ALD precursors are capable of this - radicals will react at the first collision with the surface and will thus be lost at the orifice of a feature. It has, however, been shown that this problem can be overcome for oxides deposited with an oxygen plasma by tuning the plasma parameters to achieve a higher density of radicals, while for the deposition of metals using a hydrogen plasma is severely hampered by hydrogen radical recombination. ${ }^{30}$

In plasma ALD both the plasma discharge and the precursor supply are pulsed to give a timeresolved process. Less common is to use a continuous plasma discharge and a pulsed precursor supply. This has, however, been demonstrated for alumina films using a continuous $\mathrm{O}_{2} / \mathrm{Ar}$ plasma and very short pulses of $\mathrm{Al}\left(\mathrm{CH}_{3}\right)_{3}$ (Fig. 6). ${ }^{31}$ The pulsing of the aluminum precursor was controlled using ALD valves ${ }^{23}$ which allowed for very short pulses and fast switching. It was found that by using a time-resolved precursor delivery into the continuous plasma discharge, a high level of control over the structural properties of $\mathrm{AlO}_{\mathrm{x}}$ could be obtained by tuning the time interval between the precursor pulses. The pulsing of $\mathrm{Al}\left(\mathrm{CH}_{3}\right)_{3}$ seemed to decouple the "film growth" and "film modification" processes that normally occur simultaneously during plasma CVD with continuous precursor flows. During the time interval between two precursor pulses, the precursor concentration changed rapidly in the plasma and 
two different plasma regimes were distinguished by monitoring the film thickness using spectroscopic ellipsometry (Fig. 6). Directly after the precursor pulse, the plasma was precursor-rich and rapid film growth took place. In the remainder of the pulse interval, the plasma operated in a precursor-depleted-regime during which film oxidation and densification primarily took place under the influence of the $\mathrm{O}_{2} / \mathrm{Ar}$ plasma. The authors concluded that the densification of the film was due to the removal of hydroxyl groups present in the film after the $\mathrm{Al}\left(\mathrm{CH}_{3}\right)_{3}$ injection rather than from ion bombardment (see below for a discussion on the effects of ion). The precursor pulse length (10-20ms) and pulse frequency were key parameters to control the growth process and to tailor the material properties. The material properties could be tuned to be comparable with $\mathrm{Al}_{2} \mathrm{O}_{3}$ synthesized by plasma $\mathrm{ALD}$, while the deposition rate was 5 times higher than for conventional plasma ALD. This is of great interest to solar cells where alumina is a potential surface passivation layer. ${ }^{32}$

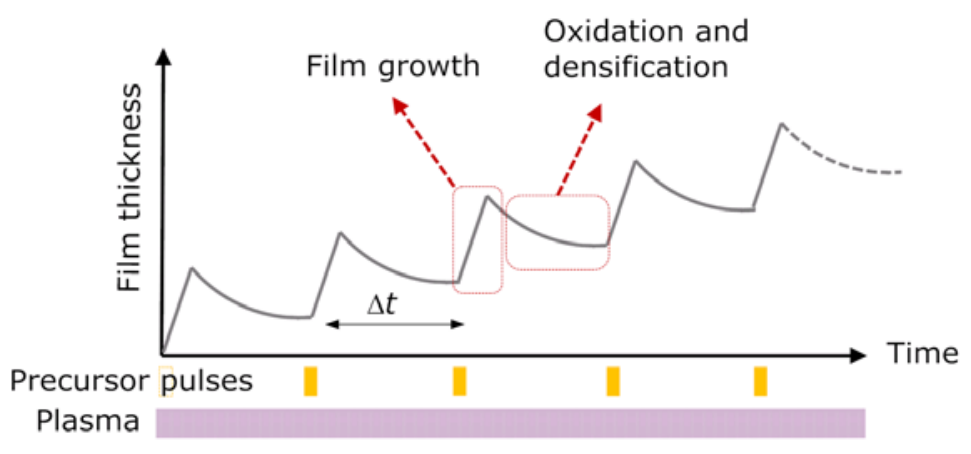

Figure 6: Schematics of the proposed film growth mechanism during precursor pulsed plasma CVD. Reprinted with permission from Ref. 31. Copyright 2012 WILEY-VCH Verlag GmbH \& Co.

\section{Time-resolved substrate bias}

A plasma is an ionized gas with freely moving electrons and ions, plasma in CVD and ALD are electropositive, meaning that the only ions positively charged. These ions are typically ionized 
atoms of the plasma gas, e.g. $\mathrm{Ar}^{+}$or $\mathrm{H}^{+}$, and they can be attracted towards the growing thin film by connecting a negative potential bias to the substrate holder. This will accelerate the positive ions toward the substrate, giving them higher energy and will lead to a bombardment of ions during film growth, which supplies more energy to the substrate surface. As mentioned above, when correctly used such a bombardment can enhance surface chemical reactions, such as ligand removal, tune the crystal phase of the film or to densify the film. Incorrect use of ion bombardment will create unwanted defects and stress in the film. It is thus a process tool that must be used with care. For more theory on substrate biasing the reader is referred to Ref. 33 .

Time-resolved substrate bias has been demonstrated in plasma ALD by connecting an RF (radio frequency) power supply to the substrate in a plasma ALD system. When the substrate stage is biased with RF power, the ion energy distribution becomes bi-modal (Fig. 7). This is because the time it takes for an ion to travel through the plasma sheath is lower than the RF cycle time (73.7 ns). Consequently, ions that pass the plasma sheath at different time instances within one RF cycle obtain different energies, since the sheath voltage drop is different. 

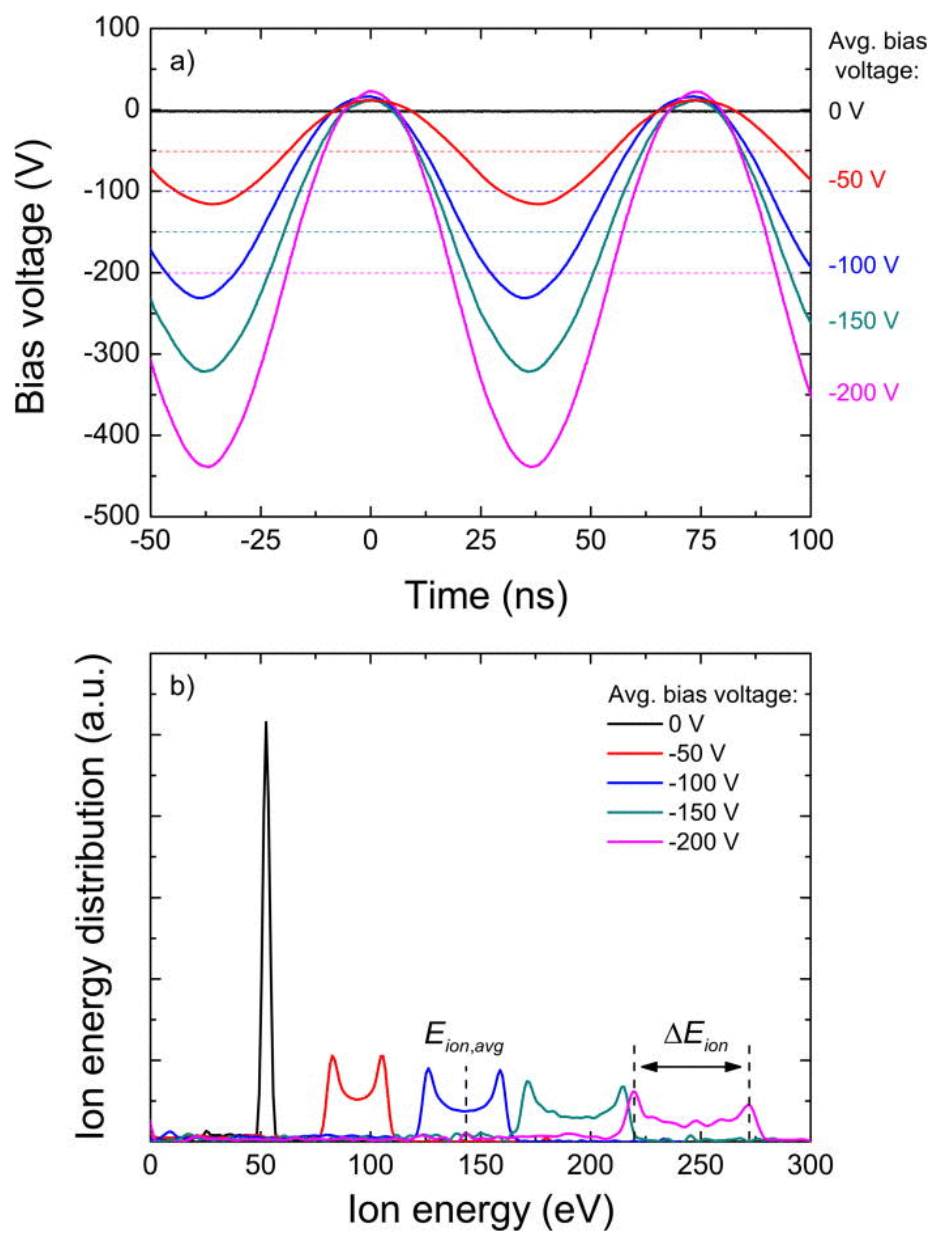

Figure 7: (a) Substrate-tuned bias voltage as a function of time and (b) corresponding ion energy distributions in $\mathrm{O}_{2}$ plasmas generated at $550 \mathrm{~W}$ ICP power and 7.5 mTorr pressure. Reprinted with permission from Ref. 33. Copyright 2013 American Vacuum Society.

By using a time-resolved substrate bias in plasma ALD between -50 to $-200 \mathrm{~V}$ and ion energies of up to $270 \mathrm{eV}$ (Fig. 7), the crystalline phase of titania deposited at $300{ }^{\circ} \mathrm{C}$ changed from anatase, when deposited without substrate bias, to rutile when a $-200 \mathrm{~V}$ substrate bias was employed. ${ }^{34}$ Deposition of alumina films by plasma ALD showed an increased growth per ALD cycle with increasing substrate bias, but also a lower film density with increasing substrate bias. The substrate bias also allowed tuning stress in the film, from tensile to compressive stress. ${ }^{33}$ $\mathrm{Co}_{3} \mathrm{O}_{4}$ films deposited by plasma ALD with applied substrate bias showed a higher mass density and a lower growth per ALD cycle with increasing substrate bias. ${ }^{33}$ 
The material properties of thin films synthesized by plasma ALD can thus be tailored by enhancing the ion energy using substrate biasing. The authors speculated ${ }^{33}, 34$ that improved control over the ion energy distribution should be beneficial to control ion- induced effects even more precisely. Here bias signals which results in narrower ion energy distributions, (e.g., signals with higher frequencies or pulsed signals) would be of interest.

\section{Time-resolved plasma discharge for self-limiting deposition chemistry}

Most of the examples of time-resolved CVD presented in this perspective were not purposefully using time as a fourth dimension to access new possibilities for film deposition. Often the processes happen to be time-resolved when - for example -precursor pulsing are done. One very interesting example where time is in fact intentionally used to achieve new features in the deposition is time-resolved plasma discharge to achieve self-limiting deposition in plasma CVD, self-limiting deposition here is defined as having a deposition rate that is independent of the duration of the individual process steps. By pulsing the plasma in a standard plasma CVD deposition process, i.e. by switching the plasma on and off, and carefully selecting the precursor chemistry, self-limiting growth similar to ALD can be achieved without pulsing the flows of precursors as is done in ALD and plasma ALD. This has been demonstrated for metal oxide films: $\mathrm{Ta}_{2} \mathrm{O}_{5}{ }^{35}, \mathrm{Al}_{2} \mathrm{O}_{3}{ }^{36}, \mathrm{ZnO}^{37}, \mathrm{TiO}_{2}{ }^{38}$, and mixed metal oxide laminates of $\mathrm{TiO}_{2}-\mathrm{SiO}_{2}{ }^{39}$ and $\mathrm{TiO}_{2}-\mathrm{Al}_{2} \mathrm{O}_{3}{ }^{40}$ and the metal sulphide $\mathrm{FeS}_{2}{ }^{41}$. This type of self-limiting deposition by plasma CVD requires two things. First, it is vital to use precursor combinations that do not react together when mixed. Oxygen gas was used as the oxygen precursor for all the metal oxide films above. The common thermal ALD oxygen precursor, $\mathrm{H}_{2} \mathrm{O}$, would be reactive with the metal precursors used; pentaetoxy tantalum $\left(\mathrm{Ta}\left(\mathrm{OC}_{2} \mathrm{H}_{5}\right)_{5}\right)$, trimethyl aluminum $\left(\mathrm{Al}\left(\mathrm{CH}_{3}\right)_{3}\right)$, dimethyl zinc $\left(\mathrm{Zn}\left(\mathrm{CH}_{3}\right)_{2}\right)$, titanium tetrachloride $\left(\mathrm{TiCl}_{4}\right)$ and silicon tetrachloride $\left(\mathrm{SiCl}_{4}\right)$. The 
sulphur precursor used is $\mathrm{H}_{2} \mathrm{~S}$ as it is not reactive with the iron precursor, iron pentacarbonyl

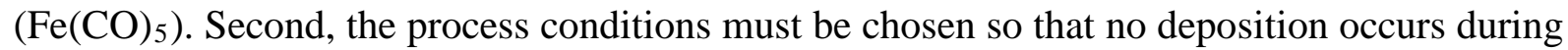
continuous plasma operation. Thus no film should be deposited without plasma and no film should be deposited with continuous plasma. The time-resolved plasma discharge is thus vital to the deposition.

The time-resolved plasma discharge is suggested to give to different process conditions (Fig. 8) which combined sequentially gives a self-limiting deposition. ${ }^{42}$ First when the plasma is OFF, the metal precursor is allowed to adsorb on the surface via self-limiting surface chemical reactions similar to ALD. And just as in plasma ALD, the second precursor $\mathrm{O}_{2}$, is present but not reactive without a plasma discharge. Thus, during plasma OFF the process essentially mimics the first step in plasma ALD. Then during plasma ON, atomic oxygen is formed from the $\mathrm{O}_{2}$ molecules which can oxidize the adsorbed metal precursors on the surface forming a monolayer of the metal oxide thin film. Contrary to plasma ALD, the metal precursor is still present in the chamber, therefore, during plasma ON the process is analogous to plasma CVD, with additional deposition occurring through plasma-activated intermediates produced at the initiation of each plasma pulse. No detailed analysis of the chemistry occurring at the start of each plasma pulse has been presented, but it is suggested that oxidation of an organometallic precursor such as $\mathrm{Al}\left(\mathrm{CH}_{3}\right)_{3}$ in the gas phase proceeds through a series of plasma chemical reactions upon plasma ignition, forming more reactive species responsible for growth in a plasma CVD mode. It is believed that oxidation of the adsorbed metal precursors takes place only during the first fraction of the plasma ON state, then as the concentration of atomic oxygen builds up, the metal precursors are fully oxidized in the gas phase and film growth steps. During the remainder of the plasma ON step the film is exposed to a high flux of $\mathrm{O}$ atoms that is presumed to be critical for film quality. ${ }^{42}$ 


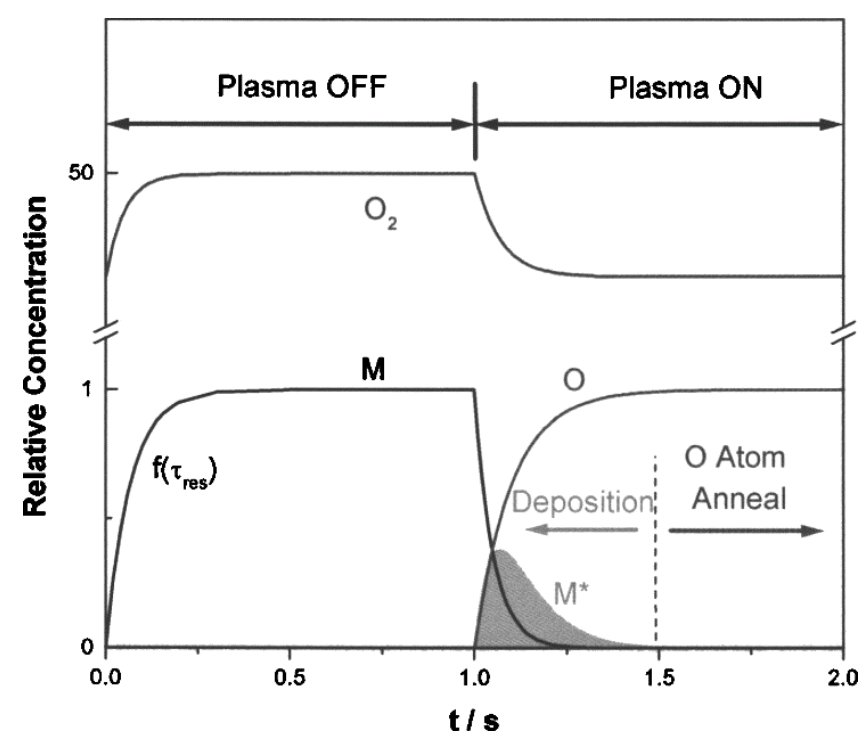

Figure 8: The concentration of metal precursor $(\mathrm{M})$ and oxygen precursor $\left(\mathrm{O}_{2}\right)$ during pulsed plasma CVD of self-limiting deposition of metal oxide films. The film deposition first occurs by ALD mode during plasma OFF and then by plasma CVD mode during the first half second of plasma ON after which the film is subjected to a flux of atomic oxygen which is believed to be beneficial for the film quality. Reprinted with permission from Ref. 43. Copyright 2007 American Vacuum Society.

In comparison to ALD and especially to plasma ALD, time-resolved, self-limiting plasma CVD offers a simpler process where there is no need to pulse gas flows nor for a purge step in the gas pulsing sequence. This allows the deposition of films in time-resolved plasma CVD with an order of magnitude higher deposition rate compared to plasma ALD while retaining a selflimiting deposition chemistry. However, ALD films are typically used in applications where only very thin films are needed, making the higher deposition rate less of an advantage. The film conformality in time-resolved plasma CVD has been shown to be nearly as good as that of plasma ALD in slightly tapered trenches with aspect ratios of 1:10. 


\section{Time-resolved plasma discharge for enhanced plasma chemistry}

A fundamental property of plasma is the plasma density, (sometimes referred to as the electron density) which is a measure on the number of free electrons in the plasma per unit volume. As the plasma chemistry is largely based on free electrons, both as reactants and for producing energetic species for subsequent reactions (Fig. 4), more plasma chemistry can be done at higher the plasma density. Free electrons in the plasma are created when the plasma gas is ionized: for cold plasmas used in plasma CVD this is typically done by an electric discharge or an applied electric field. A higher plasma density requires then that a higher amount of energy is supplied to the discharge, given that the ionization potential of the plasma gas is fixed. However, when trying to achieve a higher plasma density, and thereby a more efficient plasma chemistry, by adding more and more power to the plasma discharge, one eventually will supply too much energy and damage expensive equipment if the discharge is continuous. If the energy supply is time-resolved, giving very high power in very short pulses, a pulsed plasma discharge with very high plasma density can be obtained. This sort of plasma discharge is the foundation for the thin film deposition technique known as HiPIMS (High Power Impulse Magnetron Sputtering)

or HPPMS (High Power Pulse Magnetron Sputtering), ${ }^{44}$ which is a form of physical vapor deposition (PVD) relying mainly on atomic vapors rather than chemical reactions. In HiPIMS 10-500 $\mu$ s electric discharges with around $1000 \mathrm{~W} \mathrm{~cm}^{-2}$ are applied to magnetrons to create plasmas with plasma densities of $10^{18}-10^{19} \mathrm{~m}^{-3}$. For comparison $10^{14}-10^{16} \mathrm{~m}^{-3}$ is a typical plasma density for plasma CVD. ${ }^{24}$ Since the high power discharge frequency is only $10-1000 \mathrm{~Hz}$ and the pulse time is on the microsecond scale, it means that the power is only delivered during a few percent of the time, i.e. the duty factor of the discharge is very low. To the naked eye the plasma discharge appears continuous but it is in fact time-resolved.

In HiPIMS the high plasma density is used to ionize the flux of atoms ejected from the metallic target mounted on the magnetron. But for a plasma CVD process a higher plasma density could 
be used to do more plasma chemistry with the precursor molecules to make better use of them or to allow for less reactive precursors. ${ }^{45}$

Plasma CVD with time-resolved high power plasma discharges has only been sparsely explored. An early report aimed to deposit high quality hard coating alumina films at lower temperatures than the $1000-1100{ }^{\circ} \mathrm{C}$ typically employed in thermal CVD of alumina. ${ }^{46}$ The challenge of depositing a hard coating alumina from $\mathrm{AlCl}_{3}$ by plasma CVD is incorporation of chlorine, which severely reduces the film toughness. By increasing the voltage in the pulsed plasma discharge, and thereby the power to the plasma discharge, an increased intensity of products from the plasma chemical reactions could be observed by optical emission spectroscopy (OES) of the plasma (Fig. 9). ${ }^{47}$

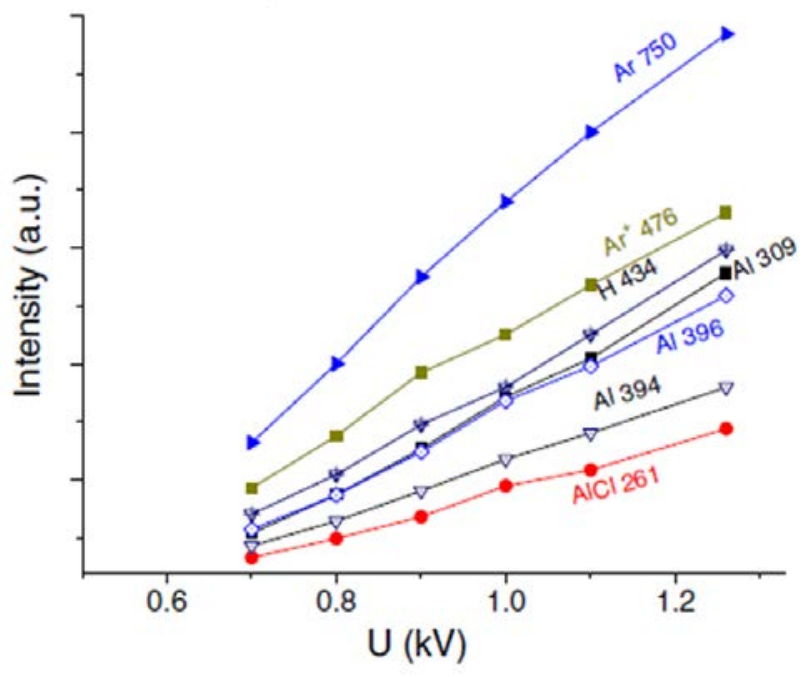

Figure 9: The intensity of emission lines from products of plasma chemical reactions as a function of the discharge voltage and thereby the power in the plasma discharge. The higher the plasma power, the higher the intensity indicating more of the species formed at higher plasma power. Reprinted with permission from Ref. 47. Copyright 2010 IOP Publishing Ltd.

The higher emission intensity recorded at higher plasma power is a strong indication that more species are formed via plasma chemical reactions at higher plasma power and thus that the 
plasma chemistry is more efficient at these higher plasma powers. By using time-resolved high power plasma discharges it was possible to obtain a plasma chemistry capable of efficiently dissociating the $\mathrm{AlCl}_{3}$ precursor to allow for deposition of phase-pure $\alpha$-alumina film at just below $600{ }^{\circ} \mathrm{C}$. The mechanical properties of the films were fully comparable to standard thermal CVD hard alumina coatings. This could thus be a low temperature CVD route to hard alumina coatings for tools which cannot tolerate high CVD temperatures due to very sharp edges.

Another report on the use of time-resolved high power plasma discharges in plasma CVD explored this very by depositing carbon films from acetylene. ${ }^{48}$ Carbon films where studied where only one precursor, a simple hydrocarbon, was used along with a plasma gas (in this case argon). It was found that a higher deposition rate was obtained from the same flow of precursor and the same amount of supplied time-averaged power when part of the power was supplied in the form of high power pulses. It was further shown that when high power pulses were used for the plasma discharge, both the plasma gas and carbon species were ionized in the plasma and thus there also was an ionic contribution to the film growth chemistry.

None of the reported studies on the use of time-resolved high power plasma discharges in plasma CVD have shown measurement of the plasma density in these processes. This because the process pressure, $65-175 \mathrm{~Pa},{ }^{45}$ has been too high for Langmuir probes ${ }^{49}$ and the collisionless plasma probe theory ${ }^{50}$ to work. In an attempt to indirectly measure the plasma density, the discharge current on the substrate holder was measured. ${ }^{51}$ The discharge current measured has been shown to directly correlate with the plasma density in HiPIMS. ${ }^{52}$ Here a higher discharge current was recorded when a higher amount of the total power was supplied as high power pulses, indicating a higher plasma density with more power supplied in high power pulses. 


\section{Outlook}

Many of the CVD methods reviewed in this perspective have, with a few exceptions, not been intentionally time-resolved but have become examples of time-resolved deposition as a consequence of some modification to the regular CVD approach. My aim with this perspective has been to show how, by carefully considering the time dimension, CVD can still evolve significantly to meet new challenges in thin film technology. The field of CVD is therefore far from complete. The question is: what can be achieved when CVD processes are time-resolved on purpose?

A recent understanding of the importance of the time dimension in plasma ALD shows that the gas residence time in the deposition chamber during the plasma step is of vital importance for controlling impurity levels in the films. ${ }^{53}$ Too long of a gas residence time, i.e. that the gas is not pumped out of the deposition chamber fast enough, allowed ligands and ligand fragments to be broken down in the plasma to deposit impurities in the film. This strongly indicates the importance of the time dimension in plasma ALD. There has also been speculation about the possibility of making plasma ALD processes intentionally more time-resolved. For example g the substrate bias signal could be ramped up or down with time, such that substrate biasing is only enabled during part of a plasma exposure step or only during every couple of cycles. Another possibility would be to decouple the physical and chemical effects of the plasma exposure step by first exposing the sample to a reactive plasma with substrate biasing disabled and subsequently exposing it to a noble gas plasma with substrate biasing enabled. ${ }^{33}$ Also, taking gas residence time into account will lead to a very carefully controlled time-resolved CVD process.

As I hope to have shown in this perspective, some very promising time-resolved plasma CVD techniques use very interesting time-resolved plasma chemistry. I believe that we have only 
seen a fraction of what can be done with well-controlled plasma chemistry. A better understanding of how a carefully chosen plasma chemistry can be controlled by time resolved plasma discharges will likely lead to several new CVD routes.

Thermal CVD also can be greatly improved by using time in a creative way. Recently a timeresolved thermal CVD route was presented where the substrate was partly covered by a shield which was removed in steps over time to uncover fresh substrate surface. ${ }^{54}$ This allowed the authors to study the evolution of a Sn-Ti-O film over time, revealing that a $\mathrm{SnO}_{2}$ film initially forms and $\mathrm{TiO}_{2}$ grows onto it, followed by Sn diffusion. Another example is the self-seeding, catalyst-free growth of gallium oxide nanostructures, demonstrated by a time-resolved CVD process. ${ }^{55}$ This process used a novel Ga precursor which was shown to produce different shapes of nanostructures when pulsed together with oxygen or water as oxidants. This sort of timeresolved CVD process shows the beauty and potential for novel types of thin film structures available to time-resolved CVD. And how can the field of ALD evolve if kinetically stable monolayers are also accessed by using kinetics as a precursor?

Most likely some very interesting CVD routes will be developed in the future when the CVD community starts to cleverly combine different types of time-resolved factors, presented in this perspective. This together with the continuous development of CVD precursor molecules ${ }^{56}$ will allow us to satisfy the need for more complex thin film materials and structures.

\section{Biography}

Henrik Pedersen received his MSc in Chemistry in 2004 and his PhD in Materials Science in 2008, both from Linköping University in Sweden. After a stint as industrial researcher at Sandvik Tooling Research and Development center in Stockholm, Sweden, he returned to academia and is today an associate professor in inorganic chemistry at Linköping University. 
He has during the last decade been exploring CVD for electronic materials, hard coatings and neutron detectors. His research is focused on understanding and developing new and better chemical vapor deposition methods.

\section{Acknowledgements}

I am very grateful to Martin Pedersen for his interpretation of this paper in the table of contents image, also given in the abstract, and to Seán Barry for critically reading and commenting on the manuscript. I also wish to express my gratitude to the editors of Chemistry of Materials for the opportunity to write this perspective.

\section{References}

(1) Pedersen, H.; Elliott, S. D. Studying chemical vapor deposition processes with theoretical chemistry. Theor. Chem. Acc. 2014, 133, 1476.

(2) Gordon, R. G.; Hausmann, D.; Kim, E.; Shepard, J. A kinetic model for step coverage by atomic layer deposition in narrow holes or trenches. Chem. Vap. Deposition 2003, 9, 73-78.

(3) Yanguas-Gil, A.; Yang, Y.; Kumar, N.; Abelson, J. R. Highly conformal film growth by chemical vapor deposition. I. A conformal zone diagram based on kinetics. J. Vac. Sci. Tech. A 2009, 27, 1235-1243.

(4) Bohr, M. T.; Chau, R. S.; Ghani, T.; Mistry, K. The High-k solution. IEEE Spectrum 2007, 44, 29-35.

(5) Leskelä, M.; Ritala, M. Atomic layer deposition (ALD): from precursors to thin film structures. Thin Solid Films 2002, 409, 138-146. 
(6) Leskelä, M.; Ritala, M. Atomic layer deposition chemistry: recent developments and future challenges. Angew. Chem. Int. Ed. 2003, 42, 5548-5554.

(7) Puurunen, R. L. Surface chemistry of atomic layer deposition: A case study for the trimethylaluminum/water process. J. Appl. Phys. 2005, 97, 121301.

(8) George, S. M. Atomic layer deposition: an overview. Chem. Rev. 2010, 110, 111-131.

(9) Miikkulainen, V.; Leskelä, M.; Ritala, M.; Puurunen, R. L. Crystallinity of inorganic films grown by atomic layer deposition: Overview and general trends. J. Appl. Phys. 2013, 113, 021301.

(10) Knoops, H. C. M.; Potts, S. E.; Bol, A. A.; Kessels, W. M. M. Atomic Layer Deposition In: Handbook of Crystal Growth: Thin Films and Epitaxy; Kuech, K. F. Ed.; Elsevier; Amsterdam, The Netherlands, 2015; pp. 1101-1134.

(11) Knisley, T. J.; Kalutarage, L. C.; Winter, C. H. Precursors and chemistry for the atomic layer deposition of metallic first row transition metal films. Coord. Chem. Rev. 2013, 257, 3222-3231.

(12) Johnson, R. W.; Hultqvist, A.; Bent, S. F. A brief review of atomic layer deposition: from fundamentals to applications. Mater. Today, 2014, 17, 236-246.

(13) Knapas, K; Ritala, M. In Situ Studies on Reaction Mechanisms in Atomic Layer Deposition. Crit. Rev. Sol. State Mater. Sci. 2013, 38, 167-202.

(14) Hersee, S. D.; Sun, X.; Wang, X. The Controlled Growth of GaN Nanowires. Nano Lett. 2006, 6, 1808-1811.

(15) Lindahl, E.; Ottosson, M.; Carlsson, J-O. Gas-Pulsed CVD for Film Growth in the CuNi-N System. Chem. Vap. Deposition 2012, 18, 10-16.

(16) Krumdieck, S. P.; Cave, H. M.; Baluti, S.; Jermy, M.; Peled, A. Expansion transport regime in pulsed-pressure chemical vapor deposition. Chem. Eng. Sci. 2007, 62, 6121-6128. 
(17) Lee, D.; Krumdieck, S.; Talwar, S. D. Scale-up design for industrial development of a PP-MOCVD coating system. Surf. Coat. Technol. 2013, 230, 39-45.

(18) Xie, H.; Raj, R. Epitaxial $\mathrm{LiTaO}_{3}$ thin film by pulsed metalorganic chemical vapor deposition from a single precursor. Appl. Phys. Lett. 1993, 63, 3146-3148.

(19) Versteeg, V. A.; Avedisian, C. T.; Raj, R. Metalorganic Chemical Vapor Deposition by Pulsed Liquid Injection Using an Ultrasonic Nozzle - Titanium Dioxide on Sapphire from Titanium(IV) Isopropoxide. J. Amer. Ceram. Soc. 1995, 78, 2763-2768.

(20) Siriwongrungson, V.; Krumdieck, S. P.; Alkaisi, M. M. Conformality investigation of titanium dioxide thin films on 3-D micrometer- and nanometer-scale features by pulsedpressure metal-organic CVD. Chem. Vap. Deposition 2011, 17, 327-336.

(21) Krumdieck, S. P.; Sbaizero, O.; Bullert, A. Raj, R. YSZ layers by pulsed-MOCVD on solid oxide fuel cell electrodes. Surf. Coat. Technol. 2003, 167, 226-233.

(22) Hartshorn, R.; Stockwell, S.; Lebedev, M.; Krumdieck, S. Precursor system for biointegration ceramics and deposition onto tantala scaffold bone interface surfaces. Surf. Coat. Technol. 2007, 201, 9413-9416.

(23) Swagelock catalog: Diaphragm valves for Atomic Layer Deposition. Available on http://www.swagelok.com/downloads/WebCatalogs/en/MS-02-301.PDF, accessed November 21, 2015

(24) Hess, W.; Graves, D. B. In Chemical Vapor Deposition: Principles and Applications; Hitchman, M. L.; Jensen, K. F., Eds.; Academic Press Ltd: San Diego, 1993, pp. 385-435. (25) Konyashin, I.; Bill, J.; Aldinger, F. Plasma-Assisted CVD of Cubic Boron Nitride. Chem. Vap. Deposition 1997, 3, 239-255.

(26) Profijt, H. B.; Potts, S. E.; van de Sanden, M. C. M.; Kessels, W. M. M. Plasma-Assisted Atomic Layer Deposition: Basics, Opportunities, and Challenges. J. Vac. Sci. Technol. A 2011, 29, 050801. 
(27) Potts, S. E.; Profijt, H. B.; Roelofs, R.; Kessels, W. M. M. Room-Temperature ALD of Metal Oxide Thin Films by Energy-Enhanced ALD. Chem. Vap. Deposition 2013, 19, 125133.

(28) Mackus, A. J. M.; Garcia-Alonso, D.; Knoops, H. C. M.; Bol, A. A.; Kessels, W. M. M. Room-Temperature Atomic Layer Deposition of Platinum. Chem. Mater. 2013, 25, 17691774.

(29) Knoops, H. C. M.; Langereis, E.; van de Sanden, M. C. M.; Kessels, W. M. M. Conformality of Plasma-Assisted ALD: Physical Processes and Modeling. J. Electrochem. Soc. 2010, 157, G241-G249.

(30) Kariniemi, M.; Niinistö, J.; Vehkamäki, M.; Kemell, M.; Ritala, M.; Leskelä, M.;

Putkonen, M. Conformality of remote plasma-enhanced atomic layer deposition processes: An experimental study. J. Vac. Sci. Technol. A 2012, 30, 01A115.

(31) Dingemans, G.; van de Sanden, M. C. M.; Kessels, W. M. M. Plasma-enhanced Chemical Vapor Deposition of Aluminum Oxide Using Ultrashort Precursor Injection Pulses. Plasma Process. Polym. 2012, 9, 761-771.

(32) Dingemans, G.; Kessels, W. M. M. Status and prospects of $\mathrm{Al}_{2} \mathrm{O}_{3}$-based surface passivation schemes for silicon solar cells. J. Vac. Sci. Technol. A 2012, 30, 040802. (33) Profijt, H. B.; van de Sanden, M. C. M.; Kessels, W. M. M. Substrate-biasing during plasma-assisted atomic layer deposition to tailor metal-oxide thin film growth. J. Vac. Sci. Technol. A 2013, 31, 01A106.

(34) Profijt, H. B.; van de Sanden, M. C. M.; Kessels, W. M. M. Substrate Biasing during Plasma-Assisted ALD for Crystalline Phase-Control of $\mathrm{TiO}_{2}$ Thin Films Electrochem. Sol. Stat. Lett. 2012, 15, G1-G3. 
(35) Seman, M.; Robbins, J.J.; Agarwal, S.; Wolden, C. A. Self-limiting growth of tantalum oxide thin films by pulsed plasma- enhanced chemical vapor deposition. Appl. Phys. Lett. 2007, 90, 131504.

(36) Szymanski, S. F.; Seman, M. T.; Wolden, C. A. Plasma and gas-phase characterization of a pulsed plasma-enhanced chemical vapor deposition system engineered for self-limiting growth of aluminum oxide thin films. Surf. Coat. Technol. 2007, 201, 8991-8997.

(37) Rowlette, P. C.; Allen, A. G.; Bromley, O. B.; Wolden, C. A. Self-limiting deposition of semiconducting ZnO by pulsed plasma-enhanced chemical vapor deposition. J. Vac. Sci. Technol. A 2009, 27, 761-766.

(38) Kubala, N. G.; Rowlette, P. C.; Wolden, C. A. Self-Limiting Deposition of Anatase $\mathrm{TiO}_{2}$ at Low Temperature by Pulsed PECVD. Electrochem. Solid Stat. Lett. 2009, 12, H259-H262. (39) Rowlette, P. C.; Wolden, C. A. Digital control of $\mathrm{SiO}_{2}-\mathrm{TiO}_{2}$ mixed-metal oxides by pulsed PECVD. ACS Appl. Mater. Interfaces 2009, 1, 2586-2591.

(40) Rowlette, P. C.; Wolden, C. A. Pulsed plasma-enhanced chemical vapor deposition of $\mathrm{Al}_{2} \mathrm{O}_{3}-\mathrm{TiO}_{2}$ nanolaminates. Thin Solid Films 2010, 518, 3337-3341.

(41) Sentman, C. D.; O’Brien, M.; Wolden, C. A. Self limiting deposition of pyrite absorbers by pulsed PECVD. J. Vac. Sci. Technol. A 2014, 32, 021201.

(42) Seman, M. T.; Richards, D. N.; Rowlette, P.; Wolden, C. A. An Analysis of the Deposition Mechanisms involved during Self-Limiting Growth of Aluminum Oxide by Pulsed PECVD. Chem. Vap. Deposition. 2008, 14, 296-302.

(43) Szymanski, S. F.; Seman, M. T.; Wolden, C. A. Effect of wall conditions on the selflimiting deposition of metal oxides by pulsed plasma-enhanced chemical vapor deposition. $J$. Vac. Sci. Technol. A 2007, 25, 1493-1499.

(44) Lundin, D.; Sarakinos, K. An introduction to thin film processing using high-power impulse magnetron sputtering. J. Mater. Res. 2012, 27, 780-792. 
(45) Lundin, D.; Pedersen, H. High Power Pulsed Plasma Enhanced Chemical Vapor Deposition: A Brief Overview of General Concepts and Early Results. Physics Procedia 2013, 46, 3-11.

(46) Ruppi, S. Enhanced performance of $\alpha-\mathrm{Al}_{2} \mathrm{O}_{3}$ coatings by control of crystal orientation. Surf. Coat. Technol. 2008, 202, 4257-4269.

(47) Jiang, K.; Sarakinos, K.; Konstantinidis, S.; Schnieder, J. W. Low temperature synthesis of $\alpha-\mathrm{Al}_{2} \mathrm{O}_{3}$ films by high-power plasma-assisted chemical vapour deposition. J. Phys. $D$ : Appl. Phys. 2010, 43, 325202.

(48) Pedersen, H.; Larsson, P.; Aijaz, A.; Jensen, J.; Lundin, D. A novel high-power pulse PECVD method. Surf. Coat. Technol. 2012, 206, 4562-4566.

(49) Morr-Smith, H. M.; Langmuir, I. The theory of collectors in gaseous discharges. Phys. Rev. 1926, 28, 727-763.

(50) Demidov, V. I.; Ratynskaia, S. V.; Rypdal, K. Electric probes for plasmas: The link between theory and instrument. Rev. Sci. Instrum. 2002, 73, 3409-3439.

(51) Lundin, D.; Jensen, J.; Pedersen, H. Influence of pulse power amplitude on plasma properties and film deposition in high power pulsed plasma enhanced chemical vapor deposition. J. Vac. Sci. Technol. A 2014, 32, 030602.

(52) Alami, J.; Gudmundsson, J. T.; Böhlmark, J.; Birch, J.; Helmersson, U. Plasma dynamics in a highly ionized pulsed magnetron discharge. Plasma Sources Sci. Technol. 2005, 14, 525531.

(53) Knoops, H. C. M.; de Peuter, K.; Kessels, W. M. M. Redeposition in plasma-assisted atomic layer deposition : Silicon nitride film quality ruled by the gas residence time. Appl. Phys. Lett. 2015, 107, 014102.

(54) Chadwick, N. P.; Sathasivam, S.; Bawaked, S. M.; Mokhtar, M.; Althabaiti, S. A.; Basahel, S. N.; Parkin, I. P.; Carmalt, C. J. The use of time resolved aerosol assisted chemical 
vapour deposition in mapping metal oxide thin film growth and fine tuning functional properties. J. Mater. Chem. A 2015, 3, 4811-4819.

(55) Pallister, P. J.; Buttera, S. C.; Barry, S. T. Self-seeding gallium oxide nanowire growth by pulsed chemical vapor deposition. Phys. Status Solidi A, 2015, 212, 1514-1518.

(56) Koponen, S. E.; Gordon, P. G.; Barry, S. T. Principles of precursor design for vapour deposition methods. Polyhedron 2015, DOI: 10.1016/j.poly.2015.08.024 
TOC image:

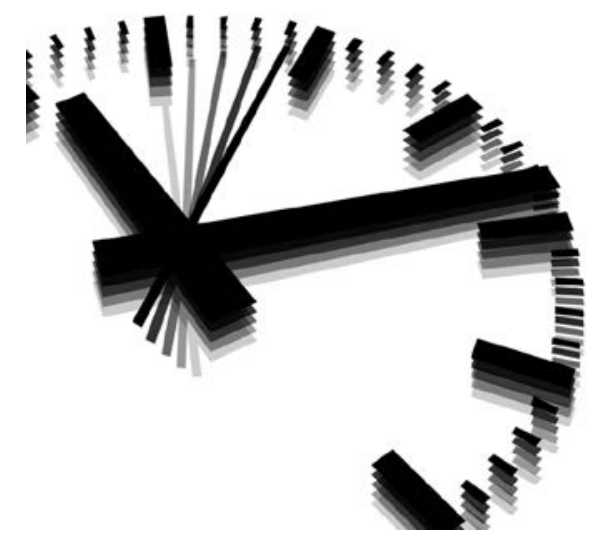

33 